\title{
Le abbreviazioni di destra e sinistra
}

\author{
Miriam Di Carlo
}

PUBBLICATO: 08 NOVEMBRE 2019

\section{Quesito:}

Alcuni lettori ci chiedono quali siano le abbreviazioni più giuste per ridurre destro/a e sinistro/a.

\section{Le abbreviazioni di destra e sinistra}

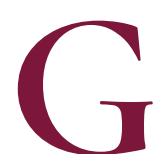

li aggettivi destro e sinistro, da cui derivano i sostantivi destra e sinistra, ricorrono di frequente nella lingua di tutti i giorni: dall'ambito medico e anatomico a quello politico, ciò che si può segmentare in due parti tra loro opposte presenta una parte destra e una sinistra. Tralasciando i sensi figurati di queste coppie di aggettivi e sostantivi (come 'propizio' e 'sfavorevole' oppure 'oriente' e 'occidente') o le estensioni di significato (come, con rinvio alla mano, 'pugno inferto con la destra o sinistra' o, nel caso di sinistro, 'evento pregiudizievole'), destro/a e sinistro/a vengono spesso usati in forma abbreviata. In questo caso si parla di abbreviazioni e non di sigle, poiché le sigle riducono sintagmi formati da più parole alle sole lettere iniziali di queste (si veda a tal proposito la scheda di Consulenza di Raffaella Setti).

Destro/a deriva dal latino dèxtěru(m) e ha riscontri in varie lingue indoeuropee, tutti collegati alla radice *dek 'ricevere' (l'Etimologico), mentre il suffisso -ter indica 'opposizione' (DELI), in questo caso con riferimento alla parte sinistra. Le abbreviazioni usate per destro/a sono:

- Dx. L'abbreviazione $d x$ comune in ambito medico, nasce per contrazione dal latino dĕxtěru(m). La forma con grafia etimologica dextro/a, ricorrente ampiamente nei testi delle origini che provengono dalle aree centrali (toscani, senesi, pisani) e settentrionali (bolognesi e veneziani), è probabilmente alla base dell'abbreviazione $d x$, registrata in molti dizionari dell'uso (GRADIT, Sabatini-Coletti 20o8, Garzanti 2017 [con la erronea indicazione di "sigla"], Zingarelli 2018) e in dizionari di sigle e abbreviazioni (Gislon-Palazzi I993, Malossini 1999, Righini 200I). Altri dizionari, anche recenti, non registrano l'abbreviazione: tra questi Devoto-Oli (sia nel lemmario che nella sez. Sigle, abbreviazioni) e Vocabolario Treccani online.

- Ds. In alcuni referti medici per 'destro/a' si può trovare $d s$, abbreviazione che contrae la forma italiana anziché la base etimologica latina. Ds viene usato con il significato di 'destro/a' anche in altri ambiti scientifici: ad esempio, consultando il corpus di Google libri si ritrova in testi riguardanti la biologia, l'architettura, l'archeologia, la filologia, in cui viene usato per indicazioni di carattere testuale. Se consideriamo il blocco di dizionari ricordati prima, l'abbreviazione ds 'destro/a' ricorre solo in Malossini 1999 e in Gislon-Palazzi 1993. Meno frequente rispetto a $d x$, DS nei testi antichi è anche abbreviazione di Deus (Mazzoleni I972); di recente è stata la sigla politica dei Democratici di Sinistra. I diversi contesti d'uso eliminano o riducono drasticamente il rischio di fraintendimenti.

- A volte vengono prodotte abbreviazioni occasionali, usate in casi sporadici e solitamente sciolte all'inizio del testo in cui ricorrono, quali des e $d r$ (quest'ultima facilmente equivocabile per dottore; cfr. la scheda di Raffaella Setti su Dott. e Dr.). Nonostante la possibile confusione, dr è registrata come abbreviazione di 'destro/a' nel Dizionario delle sigle e delle abbreviazioni di Tramonti e viene inserito con questa accezione in alcuni testi ritrovati in Google libri. 
Tirando le somme, la più comune riduzione di destro/a (registrata nella maggior parte dei dizionari dell'uso) è $d x$, come si evince dalla seguente tabella che riproduce il numero di occorrenze nelle pagine in lingua italiana su Google (ricerca effettuata il I4/6/2019, inserendo il sintagma tra virgolette del tipo "sost. + dx/ds"):

\begin{tabular}{|c|c|c|}
\hline & $\mathbf{d x}$ & ds \\
\hline Fianco & 31.500 & 1.270 \\
\hline Occhio & 16.700 & 699 \\
\hline Ginocchio & 32.500 & 2.270 \\
\hline Gamba & 30.600 & 1.300 \\
\hline Spalla & 22.100 & 4.120 \\
\hline Mano & 68.700 & 10.000 \\
\hline Braccio & 32.900 & 567 \\
\hline Piede & 29.200 & 625 \\
\hline Fanale & 34.700 & 214 \\
\hline Specchietto & 124.000 & 715 \\
\hline Retrovisore & 199.000 & 722 \\
\hline Faro & 110.000 & 555 \\
\hline Freno & 134.000 & 4.910 \\
\hline
\end{tabular}

Sinistro/a deriva dal latino sinistru(m), propr. 'differente dal destro' (DELI), col suffisso -ter che ha valore oppositivo, in rapporto a dexter. La sinistra è considerata, in confronto alla destra, la mano inferiore, meno abile, più facile a stancarsi da cui il significato di 'mano vecchia' (Nocentini-Parenti). Le abbreviazioni usate per sinistro/a sono principalmente tre:

- Sx. La $x$ non etimologica sembrerebbe formata, in maniera analogica, sul modello di $d x$.

Nel latino medievale (Isidoro di Siviglia) e in alcuni testi delle origini (i Quindici segni del giudizio di Anonimo pisano [I270-9o], la marchigiana Giostra delle virtu e dei vizi e il Lucidario pisano [entrambi del XIII sec.], la siciliana Sposizione della Passione di San Matteo [I373]) ricorrono le forme sinixtra /-u, analogiche a dextra. Ecco alcuni esempi.

Dextra vocatur dando, [...] Sinixtra autem vocata quasi sine dextra, sive quod rem fieri sinat. A sinendo enim sinixtra est noncupata. (Isidoro di Siviglia, Etimologie o Origini a cura di Claudio Leonardi, Novara, UTET, 2013)

D. Che cosa est la provedensa di Dio? M. Quella congnoscensa unde Elli sae e vede tucte quelle cose che a dextra et a sinixtra. D. che est a dire ciò? M. Sinixtra si esta la terra et tucte quelle cose che suso vi sono, dextra sì est lo cielo et tucte le vertude che dentro vi sono. [...] Et però dice la Scriptura che -1 giudicio si farae in de la valle, ciò est in questo seculo, et farano li angeli per lo comendamento del Nostro Singnore di tucta la gente del mondo due parte, una a dextra et l'altra a sinixtra: quelli de la dextra serano in aire sì come angeli et quelli de la sinixtra serano in terra sì come becci. [...] D. Come dovemo noi intendere dextra et sinixtra? M. Dextra sì est la sua gloria, sinixtra sì est la terra. (Lucidario pisano, Corpus OVI)

Sinixtra ricorre anche nelle Prediche di Savonarola, nei testi autografi di Machiavelli e Guicciardini nonché in molti testi del XVI secolo. Le molteplici attestazioni di forme con $x$ pseudoetimologica potrebbero aver favorito la coniazione dell'abbreviazione $s x$. Per quanto riguarda la presenza nei dizionari, $s x$ ricorre in GRADIT, in Sabatini-Coletti 2008, in Garzanti 2017 (in questo caso come abbreviazione), in Zingarelli 20I8, nel Dizionario di Enrico Righini 200I; è assente in Gislon-Palazzi I993, in Malossini 1999 e nei dizionari (prima ricordati) che non registrano $d x$. 
- Sn. L'abbreviazione $s n$ 'sinistro/a' viene usata in unione sia con $d x$ che con $d s$. Nel primo caso $(d x / s n)$ la coppia fa riferimento alla corretta base etimologica latina di entrambe le parole, nel secondo caso $(d s / s n)$ si hanno due abbreviazioni delle forme italiane (destro/a e sinistro/a), costruite, come avviene non di rado, con le prime due consonanti delle parole. La forma sn ricorre in un articolo di "Repubblica" quale indicazione stradale, mentre le altre occorrenze riguardano una serie di lettere inviate dai lettori alle varie rubriche del giornale.

Eccone gli esempi:

Dal Parco dei Renai di Signa si prende l'argine destro (Sn orografica) per arrivare fino al ponte di San Mauro a Signa. Passato il ponte, grazie a delle scalette, si passa sull' argine opposto (Dx orografica). [...]Si scende a Dx nel centro della frazione, dalle antiche origini medievali. Superata la sede della Misericordia, subito a Sn entriamo nel nucleo tramite via Gora ed arriviamo alla chiesa dei santi Quirico e Giulitta. (Giangranco Bracci, Lungo l'argine del Bisenzio gli antichi castelli della Piana, Repubblica.it, sez. Firenze, 7/7/2007)

Caro Direttore / [...] E' [sic] mai possibile che anche su fatti cosi' dolorosi [sic] questi sciacalli (didx e sn) non perdano l'occasione per cercare di mettersi in mostra e noi ci si possa difender solo con il telecomando? (lettera di Antonio Angelotti in Falso in Bilancio, Lettere al Direttore a cura di Vittorio Zucconi, Repubblica.it, 26/II/2007)

Buongiorno, ho 50 anni, sono ex giocatore dilettante di basket, vorrei un consiglio per un eventuale intervento al ginocchio sn. affetto da seri problemi. [...] Cito di seguito la risposta del radiologo che ha effettuato gli esami richiesti dal chirurgo: "Condizione di gonartrosi bilaterale con deformazione delle spine intercondiloidee. Artrosi femoro rotulea di grado elevato con segni di iperpressione della rotula più marcata a sn. (lettera di Anonimo in Lesione della cartilagine, Ortopedia a cura di Ferdinando Priano, Repubblica.it, sez. Salute, 23/2/2010)

Nei dizionari consultati (GRADIT, Sabatini-Coletti 2008, Zingarelli 20I8, Righini 200I e GilsonPalazzi 1993) sn può abbreviare diverse parole. Se puntato, s.n. può significare 'senza numero' o 'senza nome'; $S N$ esn rinviano a Senegal (anche come targa delle auto); in chimica, nella forma $S n$, è il simbolo dello 'stagno'; in anatomia può significare 'Sistema Nervoso' nonché 'subnormale'. Come già visto per $d s$, anche sn presenta dunque una notevole variazione d'uso, che a volte può generare ambiguità.

- Sin. Altra abbreviazione usata per sinistro/a è sin, anche puntato: sin. In quest'ultima forma ricorre in Zingarelli 20I8; in GRADIT è abbreviazione di sinonimo e simbolo matematico di seno, vale solo seno in Garzanti 2017.

La tabella che segue (costruita secondo le modalità indicate in precedenza) riporta i numeri di occorrenze delle abbreviazioni per sinistro/a nelle pagine in lingua italiana su Google (tenendo conto che sin è anche forma tronca di sino nelle locuzioni sin che, sin d'ora, ecc. Di conseguenza in questo caso i totali andrebbero diminuiti, ma non è possibile precisare di quanto). 


\begin{tabular}{r|r|r|r|} 
& sx & sin & sn \\
\hline Fianco & 25.800 & 5.720 & $58 \mathrm{I}$ \\
\cline { 2 - 4 } Occhio & 16.100 & 4.030 & 1.040 \\
\cline { 2 - 4 } Ginocchio & 21.900 & 1.670 & 1.730 \\
\cline { 2 - 4 } Gamba & 31.600 & 4.380 & 2.520 \\
\cline { 2 - 4 } Spalla & 23.700 & 5.800 & 6.330 \\
\cline { 2 - 4 } Mano & 59.600 & 25.500 & 6.490 \\
\cline { 2 - 4 } Braccio & 32.700 & 9.340 & 3.150 \\
\cline { 2 - 4 } Fanale & 24.900 & 178 & 293 \\
\cline { 2 - 4 } Specchietto & 108.000 & 2.160 & 2.650 \\
\cline { 2 - 4 } Retrovisore & 228.000 & 5.200 & 672 \\
\cline { 2 - 4 } Faro & 98.600 & 3.170 & 2.270 \\
\cline { 2 - 4 } Freno & 52.900 & 6.650 & 314 \\
\cline { 2 - 4 } & & &
\end{tabular}

Il seguente grafico ottenuto con Ngram viewer di Google confronta le percentuali di occorrenze delle coppie $d x$ e sx, $d x$ e sn e $d x$ e sin nel corpus di Google Libri a partire dal I950.

\section{Google Books Ngram Viewer}

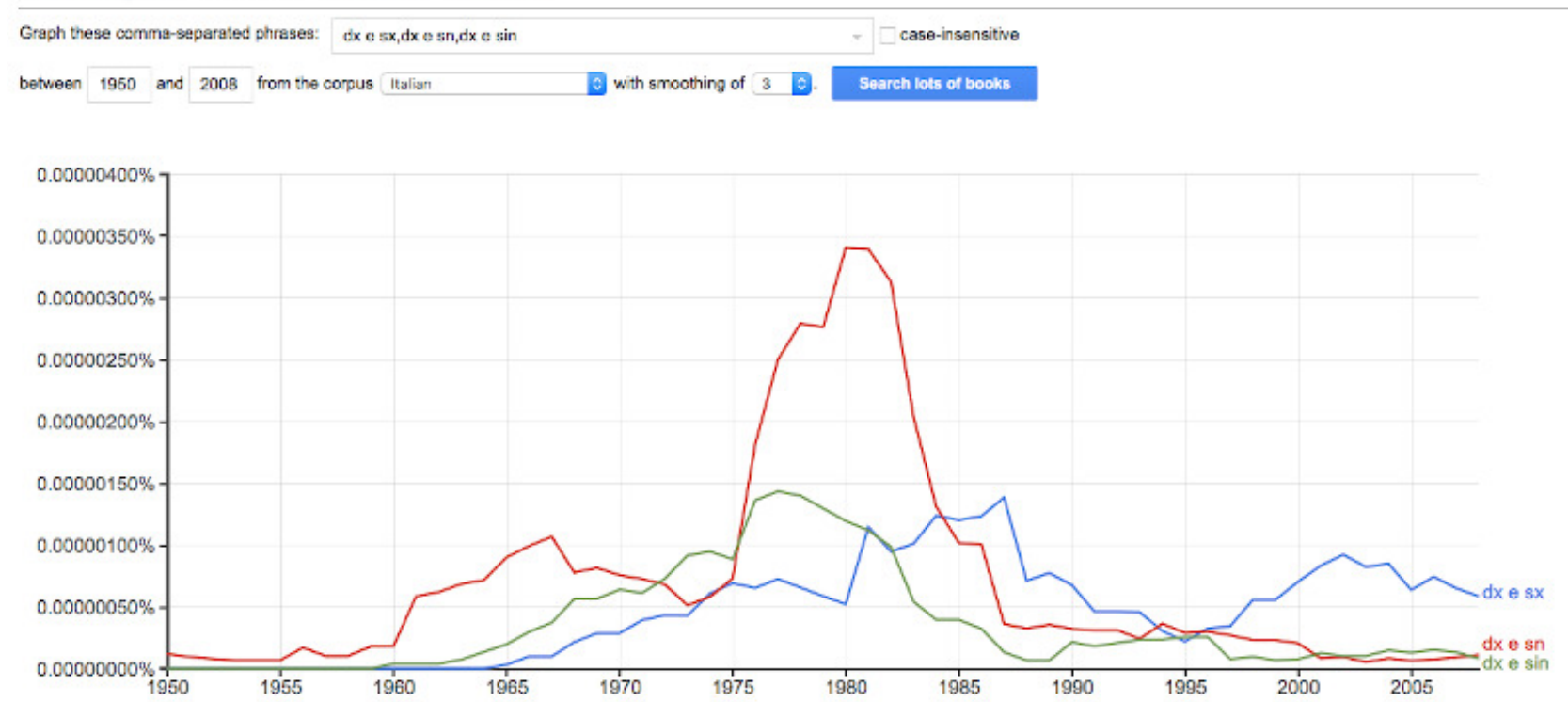

La coppia di abbreviazioni oggi prevalente in libri, giornali, rete è $d x / s x$; in ambito politico, hanno un certo successo anche le forme $c d x$ per 'centrodestra' e cs $x$ per 'centrosinistra'. Nei primi anni ' 70 invece la coppia con maggiori occorrenze è $d x / \sin$, mentre nel ventennio 1975 -1995 circa la coppia $d x / s n$ conosce una forte impennata e una altrettanto rapida discesa, le cui cause sono difficili da determinare.

Per concludere, l'abbreviazione più diffusa e conosciuta per destro/a è senza dubbio $d x$, nonostante in campo medico sia invalsa la tendenza a usare spesso $d s$. Per sinistro/a i dizionari preferiscono $s x$, non solo in ambito medico ma anche meccanico, politico, informatico, sportivo, archeologico, architettonico e nelle indicazioni stradali. L'abbreviazione sn viene usata per abbreviare altre parole con significati molto distanti tra di loro (soprattutto in ambito medico, sia nei testi specialistici destinati agli esperti del settore, sia nei testi diagnostici destinati ai pazienti), prestandosi dunque a forte ambiguità. Infine $\sin$ (anche puntato sin.) risulterebbe essere più frequente rispetto a sn.

In definitiva la coppia oggi maggiormente usata, e meno ambigua nel suo scioglimento, risulta essere $d x / s x$. 
Nota bibliografica:

- Mary Buckwell Gislon, Rosetta Palazzi, Dizionario delle sigle, Milano, Sonzogno, 1993.

- Paolo D’Achille, Fenomeni di riduzione, in Litaliano contemporaneo, Bologna, Il Mulino, 2003, pp. I52-I54.

- Ralph De Sola, Dean Stahl, Karen Kerchelich, Abbreviations dictionary, Boca Raton, CRC Press, I995.

- Andrea Malossini, Dizionario delle sigle e degli acronimi, Milano, Antonio Vallardi, 1999.

- Jole Mazzoleni, Paleografia e Diplomatica, Napoli, Libreria Scientifica Editrice, r972.

- Anna Maria Thornton, Riduzione, in Maria Grossmann, Franz Rainer (a cura di), La formazione delle parole in italiano, Tübingen, Niemeyer, 2004, pp. 555-566.

- Enrico Righini, Didisi: dizionario di Sigle, Abbreviazioni e Simboli, Bologna, Zanichelli, 200

- Nino Tramonti, Dizionario delle sigle e delle abbreviazioni, Busto Arsizio, s.e., I957.

\section{Cita come:}

Miriam Di Carlo, Le abbreviazioni di destra e sinistra, "Italiano digitale", 2019, XI, 2019/4 (ottobredicembre)

DOI: $10.35948 / 2532-9006 / 2020.3256$

\section{Copyright 2019 Accademia della Crusca}

Pubblicato con licenza creative commons CC BY-NC-ND 